\title{
Seismic signatures of fractured porous rocks: The partially saturated case
}

\author{
Santiago G. Solazzi ${ }^{1}$, Jürg Hunziker ${ }^{1}$, Eva Caspari ${ }^{1,2}$, J. Germán Rubino ${ }^{3}$, \\ Marco Favino $^{1}$, and Klaus Holliger ${ }^{1,4}$ \\ ${ }^{1}$ Institute of Earth Sciences, University of Lausanne, Lausanne, Switzerland. \\ ${ }^{2}$ Chair of Applied Geophysics, University of Leoben, Leoben, Austria. \\ ${ }^{3}$ CONICET, Centro Atómico Bariloche - CNEA, San Carlos de Bariloche, Argentina. \\ ${ }^{4}$ School of Earth Sciences, Zhejiang University, Hangzhou, China.
}

\section{Key Points:}

- We present a novel study on mesoscopic fluid-pressure diffusion effects in complex partially saturated fractured rocks.

- Attenuation and dispersion can increase for P-waves but always decrease for Swaves when a fracture network becomes partially saturated.

- We identify a novel fluid-pressure diffusion process occurring between brine- and $\mathrm{CO}_{2}$-saturated regions of a fractured network. 


\section{Abstract}

Seismic attenuation and phase velocity dispersion due mesoscopic fluid-pressure diffusion (FPD) have received increasing attention due to their inherent sensitivity to the hydromechanical properties of monosaturated fractured porous media. While FPD processes are directly affected by key macroscopic properties of fractured rocks, such as, fracture density and fracture connectivity, there is, as of yet, a lack of comprehension of the associated characteristics when multiple immiscible phases saturate the probed fractured medium. In this work, we analyze the variations experienced by $\mathrm{P}$ - and S-wave attenuation and phase velocity dispersion when $\mathrm{CO}_{2}$ percolates into an initially brine-saturated fractured porous rock. We study such variations considering a simple model of a porous rock containing intersecting orthogonal fractures as well as a more complex model comprising a fracture network. In the latter, we simulate the flow of a $\mathrm{CO}_{2}$ plume into the medium using an invasion percolation procedure. Representative samples are subjected to numerical upscaling experiments, consisting of compression and shear tests, prior to and after the $\mathrm{CO}_{2}$ invasion process. Results show that fracture-to-background FPD is only sensitive to the presence of $\mathrm{CO}_{2}$, which decreases its effects. However, fracture-tofracture FPD depends on both the overall $\mathrm{CO}_{2}$ saturation and the fluid distribution within the fracture network. While the former modulates the magnitude of the dissipation, the latter can give rise to a novel FPD process occurring between $\mathrm{CO}_{2}$-saturated and brinesaturated regions of the fracture network.

\section{Introduction}

Detecting the presence of immiscible fluid phases and monitoring their displacements throughout the subsurface by means of non-invasive geophysical techniques is widely considered to be a key frontier in the overall field of applied and environmental geophysics. New advances in this direction are of interest, for example, to $\mathrm{CO}_{2}$ geosequestration (e.g., Arts et al., 2004), geothermal energy exploitation (e.g., Farina et al., 2019), nuclear waste storage (e.g., Smith \& Snieder, 2010), and hydrocarbon exploration and production (e.g., Lumley, 2001). Seismic methods are particularly valuable for addressing these problems, as seismic waves are highly sensitive to changes in the hydraulic and mechanical properties of rocks.

Fractures are ubiquitous throughout the Earth's upper crust and, thus, they are particularly pertinent in this context (e.g., Bonnet et al., 2001). Seismic characteriza- 
tion of fractured rocks is challenging because, in most cases, the resolution of seismic data is too low for directly imaging individual fractures. Consequently, most related research efforts focus on understanding the link between fracture network characteristics and seismic attributes, such as, amplitude-variation-with-offset (AVO) (e.g., Rueger \& Tsvankin, 1997; Hunt et al., 2010; Barbosa et al., 2020). In particular, seismic attenuation and phase velocity dispersion are receiving increasing attention due to their inherent sensitivity to key macroscopic properties of fractured rocks, such as, fracture density and fracture connectivity. Effective medium approaches are commonly employed to obtain these attributes by means of analytical (e.g., Chapman, 2003, 2009; Gurevich et al., 2009) or numerical models (e.g., Rubino et al., 2013, 2014; Vinci et al., 2014). Whenever a seismic wavefield travels through a monosaturated porous fractured rock, wave-induced fluid flow (WIFF) or, as referred herein, fluid pressure diffusion (FPD) processes, play a predominant role in determining the phase velocity and amplitude decay of the waves. In presence of connected mesoscopic fractures, two manifestations of FPD can arise (Rubino et al., 2013). One is governed by FPD between compliant fractures and their stiffer embedding background, which is referred to as fracture-to-background (FB) FPD. The other manifestation is produced by FPD between connected fractures, and it is referred to as fractureto-fracture (FF) FPD. These processes have been thoroughly analyzed considering fractured rocks saturated by a single fluid phase (e.g., Rubino et al., 2013; Caspari et al., 2016; Rubino et al., 2017). However, there is a lack of comprehension of the changes that these FPD processes, and the associated effective seismic response of the medium, undergo in presence of a second immiscible saturating fluid phase with contrasting compressibility.

To date, very little work has been done with regard to the interpretation of seismic signatures of partially saturated fractured rocks. Brajanovski et al. (2010) proposed a superposition model accounting for mesoscopic FPD effects associated with partial saturation and FB fluid flow. This model considers that the background contains a patchy distribution of fluids and, also, hosts periodic monosaturated and aligned fractures. Even though this work presents the first attempt to model the seismic signatures of partially saturated fractured rocks, the considered fluid distribution is not realistic. Fluid distributions resulting from multiphase flow processes are strongly dependent on the hydraulic properties of the host rock (e.g., Rubino \& Holliger, 2012; Ba et al., 2017; Solazzi et al., $2017,2019)$. In this context, fractures constitute paths of comparatively low capillary 
resistance and, thus, non-wetting fluid phases, such as $\mathrm{CO}_{2}$, have a tendency to saturate them rather than the embedding porous background, which, in turn, tends to remain saturated with the wetting phase (e.g., brine). Taking this characteristic into account, Kong et al. (2013) studied the seismic response of rocks composed of a porous background saturated with water and permeated by a set of planar aligned fractures. The latter are saturated by a mixture of fluids, whose physical properties are modeled using an effective fluid phase. They observe that FB-FPD effects, as defined in monosaturated conditions, can be suppressed or even reversed depending on the compliance of the effective fluid saturating the fractures. The works of Amalokwu et al. $(2014,2015)$ and, more recently, of Han et al. (2019), experimentally explore the effects of partial saturation on the seismic signatures of rocks containing aligned penny shaped cracks. These studies demonstrate that ultrasonic attenuation and phase velocity, as well as anisotropy, are sensitive to the saturation state. Jin et al. (2018) proposed an approach that combines the models of Chapman (2003) and Papageorgiou and Chapman (2017) to model the behavior of S-waves observed by Amalokwu et al. (2014). They consider a collection of microscopic ellipsoidal cracks and spherical pores, which are connected with a set of mesoscale perfectly aligned fractures. All inclusions are considered to be partially saturated in a uniform manner. All of the the above mentioned models exhibit two main drawbacks. First, the effects of partial saturation on FF-FPD have not been addressed. Second, the fluids are assumed to be uniformly distributed within the fractures, even though evidence shows that fractured rocks exhibit heterogeneous fluid saturation patterns determined by the capillary pressure characteristics (e.g., Hardisty et al., 2003; Karpyn et al., 2007). Therefore, further research is needed regarding the effects of FF-FPD, in general, and of more realistic fracture networks and fluid distributions, in particular, to understand the physical processes that dominate the seismic signatures of partially saturated fractured rocks.

In this work, we analyze seismic attenuation and phase velocity dispersion as functions of frequency and incidence angle in partially saturated rocks containing interconnected stochastic fracture networks. Using an upscaling procedure based on Biot's poroelasticity equations (Rubino et al., 2016), we explore the characteristics of FB- and FFFPD processes considering an initially brine-saturated fractured formation that experiences the emplacement of $\mathrm{CO}_{2}$ along its fractures. We first consider a simple case study consisting of a low porosity rock containing a set of orthogonal and connected fractures. 
Then, a more realistic model is considered, comprising a 2D stochastic anisotropic fracture network, which contains a preferential flow path. In this case, the emplacement of $\mathrm{CO}_{2}$ is simulated considering an invasion percolation procedure.

\section{Governing Equations and Numerical Approach}

In this section, we briefly outline the upscaling procedure of Rubino et al. (2016) for anisotropic 2D media, which permits to extract the frequency- and angle-dependent P- and S-wave phase velocities and inverse quality factors for a given porous medium containing mesoscopic-scale heterogeneities. We also describe the basis of the invasion percolation (IP) procedure (Masson \& Pride, 2014; Masson, 2016), which is employed to simulate the invasion of a $\mathrm{CO}_{2}$ plume into an initially brine-saturated fractured rock.

\subsection{Biot's Consolidation Equations}

Whenever a seismic wave travels through a saturated porous rock that contains mesoscopic scale heterogeneities with contrasting compresibilities, FPD processes play a key role in the dissipation of the energy of the wave. In this context, inertial effects can usually be neglected and the medium can be correctly characterized by locally solving Biot's consolidation equations (Biot, 1941), which, in the space-frequency domain, are given by

$$
\begin{gathered}
\nabla \cdot \boldsymbol{\sigma}=0, \\
\nabla p_{f}=-i \omega \frac{\eta}{\kappa} \mathbf{w},
\end{gathered}
$$

where $\sigma$ represents the total stress tensor, $p_{f}$ is the pressure of the fluid, $\eta$ the fluid viscosity, $\kappa$ the permeability, $\omega$ the angular frequency, and $\mathbf{w}$ the relative fluid-solid displacement.

Equations (1) and (2) are coupled through the stress-strain constitutive relations (Biot, 1962)

$$
\begin{gathered}
\boldsymbol{\sigma}=2 \mu_{m} \boldsymbol{\epsilon}+\boldsymbol{I}\left(\lambda_{c} \nabla \cdot \mathbf{u}-\alpha M \zeta\right), \\
p_{f}=-\alpha M \nabla \cdot \mathbf{u}+M \zeta,
\end{gathered}
$$

where $\boldsymbol{I}$ is the identity matrix, $\mathbf{u}$ the solid displacement, and $\zeta=-\nabla \cdot \mathbf{w}$ a measure of the local change in the fluid content. The strain tensor is given by $\boldsymbol{\epsilon}=\frac{1}{2}\left(\nabla \mathbf{u}+(\nabla \mathbf{u})^{\mathrm{T}}\right)$, with the superscript $\mathrm{T}$ denoting the transpose operator. The poroelastic Biot-Willis pa- 


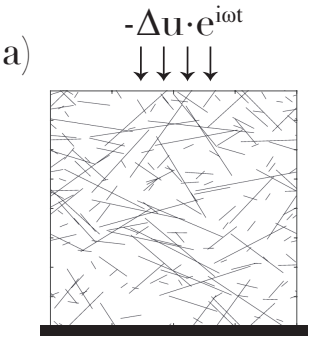

b)

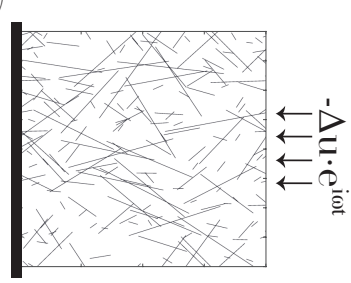

c)

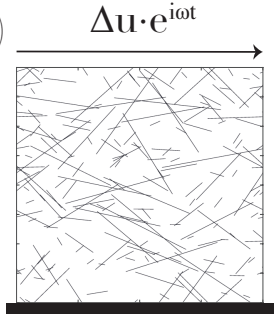

Figure 1. Schematic illustration of the (a) vertical, (b) horizontal, and (c) shear numerical oscillatory relaxation tests employed to obtain the equivalent stiffness matrix of the explored medium.

rameter $\alpha$, the fluid storage coefficient $M$, and the Lamé parameter $\lambda_{c}$ are given by

$$
\begin{gathered}
\alpha=1-\frac{K_{m}}{K_{s}}, \\
M=\left(\frac{\alpha-\phi}{K_{s}}+\frac{\phi}{K_{f}}\right)^{-1},
\end{gathered}
$$

and

$$
\lambda_{c}=K_{m}+\alpha^{2} M-\frac{2}{3} \mu_{m},
$$

with $\phi$ denoting the porosity, $\mu_{m}$ the shear modulus of the bulk material, which is equal to that of the dry frame, and $K_{f}, K_{s}$, and $K_{m}$ the bulk moduli of the fluid phase, the solid grains, and the dry matrix, respectively.

\subsection{Numerical Upscaling Procedure}

In order to obtain the seismic response, we locally solve equations (1) to (4) for a representative elementary volume (REV) of the formation of interest by means of a finite element method (Favino et al., 2019). We employ a set of boundary conditions, which can be classified in the form of two compressional and one shear oscillatory relaxation tests (Figure 1). The two compressional tests are performed by applying (1) a time-harmonic homogeneous vertical displacement at the top boundary and a null vertical displacement at the bottom of the sample (Figure 1a); and (2) a time-harmonic horizontal displacement at a lateral boundary and a null displacement at the opposite lateral boundary (Figure 1b). The third test is a simple oscillatory shear test (Figure 1c). In all tests, we apply periodic boundary conditions for the solid displacement and the fluid pressure along the remaining boundaries of the sample. Anti-periodic boundary conditions are considered for the traction and the fluid flux (Favino et al., 2019). 
The procedure is based on the assumption that the upscaled response of a poroelastic medium can be represented by an effective homogeneous viscoelastic solid (e.g., Solazzi et al., 2016). Hence, the average stress and strain fields of the sample resulting from each test are assumed to be related by an equivalent frequency-dependent complexvalued 2D stiffness matrix, which, in Voigt notation is given by (Rubino et al., 2016)

$$
\left(\begin{array}{c}
\left\langle\sigma_{11}(\omega)\right\rangle \\
\left\langle\sigma_{22}(\omega)\right\rangle \\
\left\langle\sigma_{12}(\omega)\right\rangle
\end{array}\right)=\left(\begin{array}{lll}
C_{11} & C_{12} & C_{16} \\
C_{11} & C_{22} & C_{26} \\
C_{16} & C_{26} & C_{66}
\end{array}\right)\left(\begin{array}{c}
\left\langle\epsilon_{11}(\omega)\right\rangle \\
\left\langle\epsilon_{22}(\omega)\right\rangle \\
\left\langle 2 \epsilon_{12}(\omega)\right\rangle
\end{array}\right),
$$

where the stiffness coefficients $C_{i j}$ describe the effective anisotropic viscoelastic behavior of the fractured poroelastic medium. The operator $\langle\cdot\rangle$ denotes the corresponding volume average. Once all the elements of the stiffness matrix have been retrieved, by means of a least squares procedure, it is possible to compute the equivalent complex, frequencyand angle-dependent wavenumber $\mathbf{k}(\omega, \vartheta)$ for P- and S-waves (Rubino et al., 2016). The phase velocity and inverse quality factor as functions of frequency and incidence angle respond to

$$
V_{j}(\omega, \vartheta)=\frac{\omega}{\Re\left\{\mathbf{k}_{j}(\omega, \vartheta)\right\}}, \quad Q_{j}^{-1}(\omega, \vartheta)=-\frac{\Im\left\{\mathbf{k}_{j}(\omega, \vartheta)^{2}\right\}}{\Re\left\{\mathbf{k}_{j}(\omega, \vartheta)^{2}\right\}},
$$

where $j=p, s$ denotes the corresponding wave propagation mode, and $\Re$ and $\Im$ denote the corresponding real and imaginary parts, respectively. A detailed description of the numerical upscaling procedure and its boundary conditions is given by Favino et al. (2019).

\subsection{Invasion Percolation Procedure}

The IP technique was originally introduced by Wilkinson and Willemsen (1983) to model the problem of one fluid displacing another one from a porous medium, but in principle it may be applied to any kind of invasion process which proceeds along a path of least resistance. The theory accurately reproduces the fluid distribution observed in the laboratory under quasi-static displacement, that is, when viscous forces are negligible with respect to capillary forces (Lenormand et al., 1988). In this work, we use an IP procedure developed by Masson and Pride (2014) and Masson (2016) to simulate a capillary-dominated invasion process, in which $\mathrm{CO}_{2}$ displaces brine from the fractures of a porous rock.

A key factor in the capillary displacement of immiscible pore fluid phases is the capillary entry pressure $p_{c}^{e}$. This parameter determines the minimum pressure difference between the two fluids (in this case $\mathrm{CO}_{2}$ and brine) needed to advance the fluid interface 
across a particular region of the porous rock and is given by the Young-Laplace equation (e.g., Bear, 1972)

$$
p_{c}^{e}=\frac{2 \gamma \cos \beta}{r_{p}},
$$

where $\gamma$ denotes the interfacial tension between the immiscible fluid phases, $\beta$ the contact angle, and $r_{p}$ the characteristic pore throat radius of the medium. During an IP drainage process, non-wetting fluid phases preferentially invade regions with small capillary entry pressures $p_{c}^{e}$ (large $r_{p}$ ). In this work, the properties of the background rock result in significantly large entry pressures compared to those of the fractures. Based on this characteristic, we assume that the flow of $\mathrm{CO}_{2}$ is confined to the fracture network.

For the IP process, the probed porous medium is discretized on a regular grid, where each cell $\Omega_{i j}$ has a local characteristic throat radius $r_{p, i j}$ and, hence, a particular entry pressure $p_{c, i j}^{e}$ (equation 10). Invasion and exit boundaries are defined, from which $\mathrm{CO}_{2}$ percolates through the medium and where brine escapes from it, respectively. No-flow conditions are applied on the remaining boundaries. The IP simulation starts with all the cells being fully saturated with brine. The algorithm thus comprises the following steps: (1) Find the brine-saturated cells that are in contact to the injection boundary and/or in contact with cells which have already been invaded with $\mathrm{CO}_{2} ;(2)$ invade the cell that has minimum entry pressure. The process is repeated until $\mathrm{CO}_{2}$ reaches the exit boundary.

\section{Numerical Analysis: Orthogonal Fracture Sets}

In the following, we analyze the first of the two scenarios proposed in this work to study the effects of partial saturation on the seismic response of fractured rocks. Let us consider an REV of a fractured medium with a side length of $40 \mathrm{~cm}$ comprising two perpendicular and intersecting fractures (Figure 2a). The fractures are modeled as highly compliant and highly porous and permeable rectangular features embedded in a much stiffer and much less porous and permeable background medium. Both fractures have an aperture $h$ of $0.5 \mathrm{~mm}$ and a length $l$ of $28 \mathrm{~cm}$. To explore the effects of partial saturation on seismic attenuation and phase velocity, we consider three different cases. In the first case, the rock is fully saturated with brine (Figure $2 \mathrm{~b}$ ). The second case assumes that the vertical fracture is saturated by $\mathrm{CO}_{2}$ and that the background and horizontal fracture are saturated with brine (Figure 2c). Finally, for the third case, both fractures are fully saturated with $\mathrm{CO}_{2}$ and the background is saturated with brine (Figure 2d). 

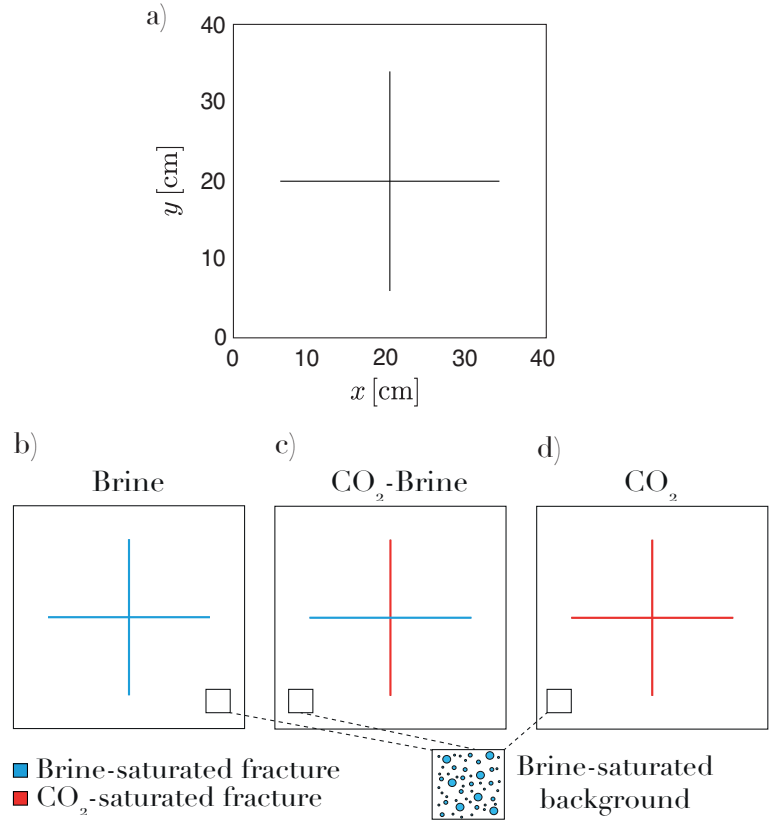

Figure 2. (a) Sample containing a pair of perpendicular intersecting fractures. We consider this simple model to analyze FPD effects in (b) fully brine-saturated and (c, d) partially saturated conditions. Note that the background embedding the fractures is fully brine-saturated for all three models.

Note that fractures may contain $\mathrm{CO}_{2}$ or brine, but we assume that the background is saturated with brine in all cases. This criterion to distribute the fluids is consistent with the fact that, given its low porosity and permeability, the background rock has a much higher capillary entry pressure than the fractures. The physical properties of the background, fractures, and pore fluids are summarized in Table 1.

The presence of brine in both background and fractures produces two FPD processes for $\mathrm{P}$-waves propagating in the vertical and horizontal directions, respectively (blue lines in Figure 3). The low- and high-frequency peaks of $Q_{p}^{-1}(\omega, \vartheta)$ are caused by FBand FF-FPD, respectively. As a consequence of the stiffening effect associated with FPD, the phase velocity $V_{p}(\omega, \vartheta)$ exhibits dispersion for those frequencies and incidence angles where FPD prevails. Due to the underlying symmetry of the medium, FF-FPD effects are negligible for $\vartheta=45^{\circ}$ (Figures $3 \mathrm{~b}$ and $3 \mathrm{e}$ ) and, thus, the high-frequency peak of the attenuation curve vanishes for this direction of propagation. When only one fracture is saturated by $\mathrm{CO}_{2}$, FB-FPD effects are weakened in comparison with the fully brinesaturated case, for all incidence angles (red lines in Figure 3). The reason for this be- 
Table 1. Rock and fluid properties. Rock properties are similar to those used by Hunziker et al. (2018), considering a less permeable background. Fluid properties are adopted from Rubino et al. (2011).

\begin{tabular}{lcc}
\hline Solid Phase & Background & Fracture \\
\hline$\kappa$ & $10^{-20} \mathrm{~m}^{2}$ & $10^{-11} \mathrm{~m}^{2}$ \\
$\phi$ & 0.05 & 0.6 \\
$K_{s}$ & $40 \mathrm{GPa}$ & $40 \mathrm{GPa}$ \\
$K_{m}$ & $37 \mathrm{GPa}$ & $4 \times 10^{-3} \mathrm{GPa}$ \\
$\mu_{m}$ & $31 \mathrm{GPa}$ & $0.02 \mathrm{GPa}$ \\
\hline Fluid Phase & $\mathbf{C O}$ & brine \\
\hline$K_{f}$ & $0.0229 \mathrm{GPa}$ & $2.3 \mathrm{GPa}$ \\
$\eta_{f}$ & $1.56 \times 10^{-5} \mathrm{~Pa} . \mathrm{s}$ & $0.001 \mathrm{~Pa} . \mathrm{s}$ \\
$\rho_{f}$ & $693 \mathrm{~kg} / \mathrm{m}^{3}$ & $1090 \mathrm{~kg} / \mathrm{m}^{3}$ \\
\hline
\end{tabular}
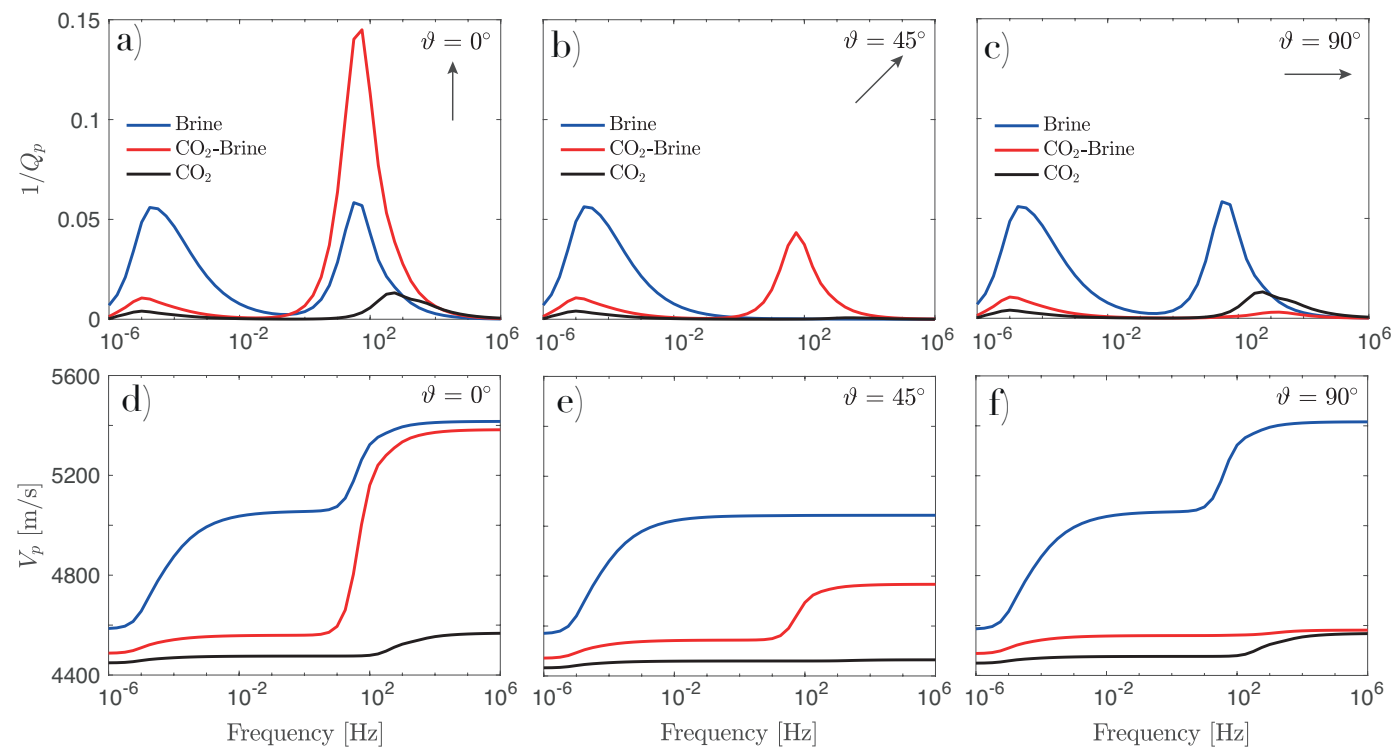

Figure 3. P-wave attenuation and phase velocity as functions of frequency for incidence angles of (a-d) $0^{\circ}$, (b-e) $45^{\circ}$, and (c-f) $90^{\circ}$, where $0^{\circ}$ and $90^{\circ}$ denote vertical and horizontal propagation, respectively. The line colors indicate the three fluid distribution scenarios illustrated in Figure 2. 

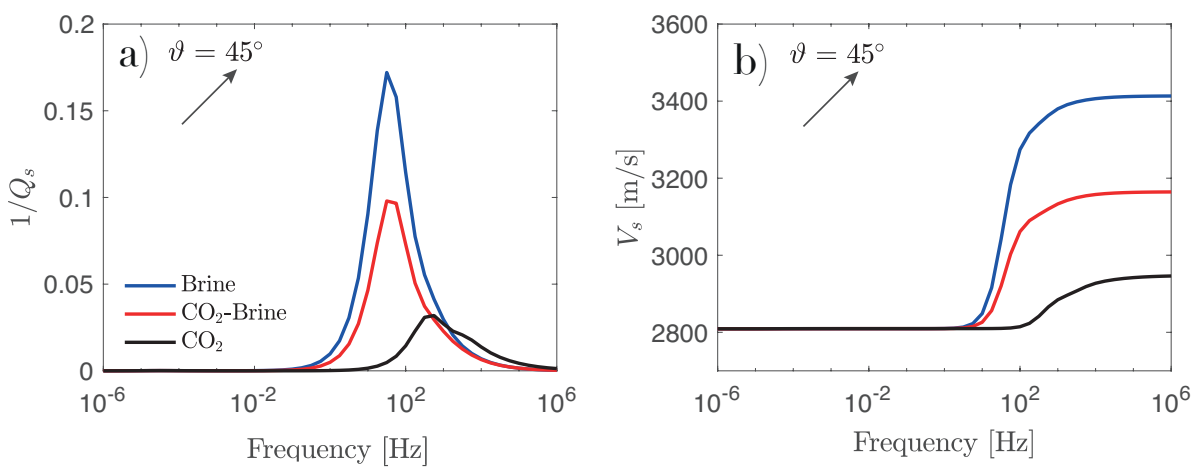

Figure 4. (a) S-wave attenuation and (b) phase velocity as functions of frequency for an incidence angle of $45^{\circ}$. The line colors indicate the three fluid distribution scenarios illustrated in Figure 2 .

havior is that FF-FPD occurs more rapidly (high frequency) than FB-FPD (low frequency). Consequently, when considering the time scales at which FB-FPD occurs, the pressure gradients within the fractures have already been equilibrated. The relaxed pressure state reached by fractures when partially saturated is lower than the one obtained when both fractures are brine-saturated, thus, resulting in smaller fluid pressure gradients with respect to the background and, also, smaller FB-FPD effects. On the other hand, FF-FPD effects are hindered or strengthened depending on the incidence angle due to the effects of partial saturation. In particular, an increase in FF-FPD effects is observed when the wave travels in the vertical direction, which is the direction of the $\mathrm{CO}_{2}$-saturated fracture (Figures 3a and 3d). Compared to the brine-saturated case, the presence of a more compliant fluid phase, such as $\mathrm{CO}_{2}$, in the vertical fracture permits a larger volume of brine to be injected from the horizontal fracture in response to the vertical compression associated with the passing seismic wave. This, in turn, results in a larger amount of energy dissipation and associated velocity dispersion. A contrasting behavior is observed for a $90^{\circ}$ incidence. Here, FF-FPD effects are much smaller than their fully brine-saturated counterparts (Figures 3c and 3f). In this case, the pressure of $\mathrm{CO}_{2}$ saturating the fracture does not evidence a significant increase as a result of the compression associated with the passing P-wave, which does not favor FPD within connected fractures. This shows that FF-FPD may be sensitive to the preferential direction of $\mathrm{CO}_{2}$ allocation in fractured networks. Finally, when the fractures are completely saturated with $\mathrm{CO}_{2}$, the seismic signatures are analogous to those of the fully brine-saturated state. However, due to the 
larger compressibility of $\mathrm{CO}_{2}$ with respect to brine, FB- and FF-FPD processes, and the associated viscous energy dissipation, are weakened (black lines in Figure 3). Please note that, when comparing the $\mathrm{P}$-wave phase velocity curves associated with the three models considered in this analysis, we observe a decrease in the corresponding values with increasing $\mathrm{CO}_{2}$ saturation (Figures 3d, 3e, and 3f).

In the presence of heterogeneities, S-waves can locally induce compression and extension, which in turn result in local variations in fluid pressure and, thus, in fluid flow (e.g., Masson \& Pride, 2007). For the considered fracture configuration, that is, two perpendicular and intersecting fractures, and considering a fully brine-saturated case, the largest S-wave attenuation due to FF-FPD is obtained for a $45^{\circ}$ incidence angle (e.g., Quintal et al., 2014). Firstly, we note that FB-FPD effects are not present in the S-wave attenuation and phase velocity dispersion curves (Figure 4). This is expected as, for such frequencies, the pressure gradients arising between the fractures have enough time to equilibrate, rendering pressure gradients between the fractures and background negligible (Quintal et al., 2014). Consequently, the attenuation associated with FB-FPD processes is virtually null for this case. Secondly, FF-FPD effects decrease when the vertical fracture is saturated with $\mathrm{CO}_{2}$ when compared with the fully brine-saturated state (Figure 4). For S-waves traveling at an incidence angle of $45^{\circ}$, there is an increase of pressure in the horizontal fractures and pressure decreases in the vertical ones (Rubino et al., 2017). In the partially saturated case (Figure $2 \mathrm{c}$ ), the pressure decrease in the $\mathrm{CO}_{2}$-saturated vertical fractures in response to a $45^{\circ} \mathrm{S}$-wave incidence is smaller than the one expected in the brine-saturated case. Thus, the pressure gradients within the fractures and the associated dissipation due to FF-FPD drops when compared to the fully brine-saturated rock. Attenuation and phase velocity dispersion values are further diminished when both fractures are saturated with $\mathrm{CO}_{2}$ (Figure 4).

An interesting aspect of the results analyzed above is that the anisotropy of the medium is changed by the presence of $\mathrm{CO}_{2}$. We note that, in the presence of partially saturated connected fractures, FB-FPD effects for P-waves decrease for all incidence angles compared with the fully brine-saturated case (Figures 5a and 5b). As previously noted, FB-FPD effects for S-waves are virtually null for both fully and partially saturated cases (Figures 5c and 5d). When analyzing the effects of partial saturation on FF-FPD effects compared to the brine-saturated case, the values for P-waves decrease for incidence angles $50^{\circ}<\vartheta<130^{\circ}$ and increase otherwise (Figures 5a and 5b). Conversely, S-wave 
a

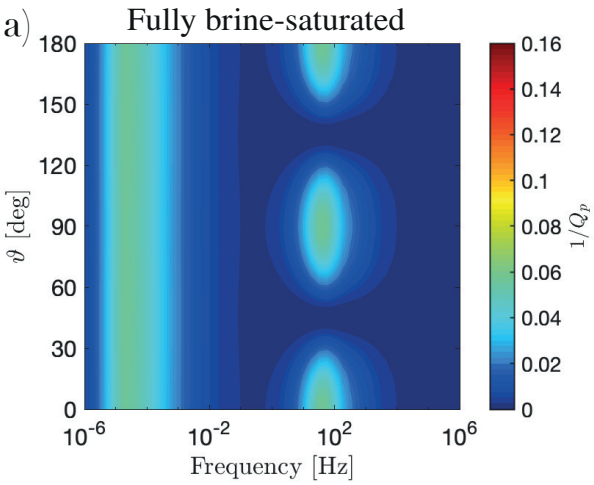

c)

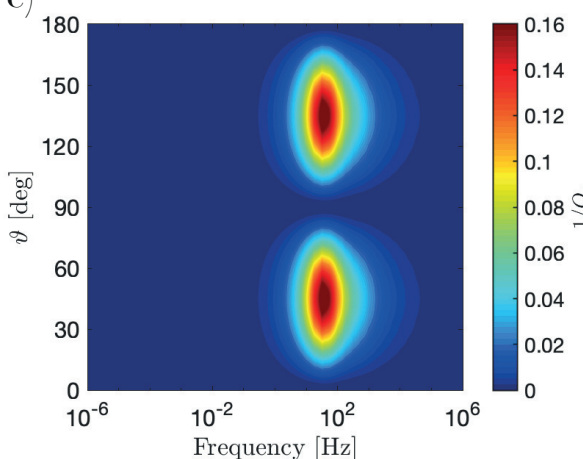

b) Brine- and $\mathrm{CO}_{2}$-saturated

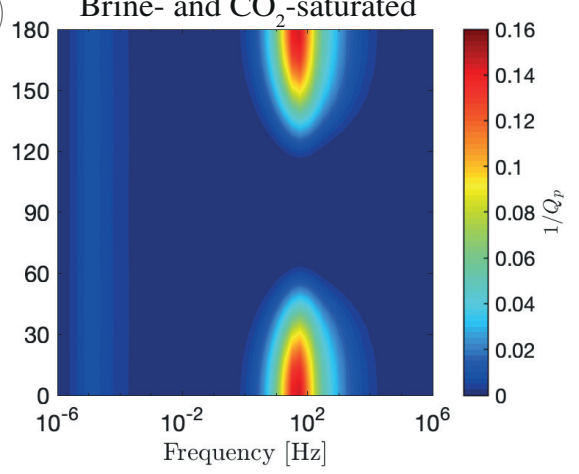

d)

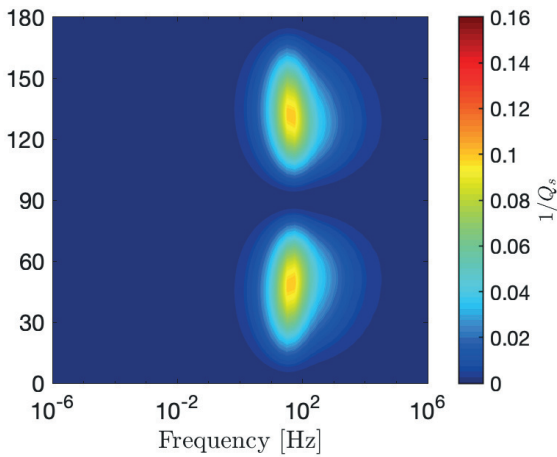

Figure 5. P-and S-wave attenuation as functions of frequency and incidence angle for the sample shown in Figure 2. Left column corresponds to the sample fully saturated with brine (Figure $2 \mathrm{~b}$ ), while the right column depicts the responses partial saturation, that is, with only the horizontal and vertical fractures saturated with brine and $\mathrm{CO}_{2}$, respectively (Figure 2c). 
attenuation due to FF-FPD decreases due to the effects of partial saturation (Figures $5 c$ and $5 \mathrm{~d})$.

These result demonstrate that the presence of a more compliant fluid phase in a restricted region of a fracture network may significantly affect the seismic attenuation and velocity dispersion characteristics which, in turn, may offer novel perspectives for the characterization for partially saturated reservoirs. To this end, we explore a more realistic scenario in the following.

\section{Numerical Analysis: Stochastic Fracture Network}

\subsection{Fracture Network and Fluid Distribution}

Let us now consider a square sample with a side length of $40 \mathrm{~cm}$ which contains a complex stochastic fracture network (Figure 6a). The fracture network is obtained following the computational procedure developed by Hunziker et al. (2018). Fracture lengths characterizing natural fracture systems are drawn from a seemingly universal power law distribution (e.g., Bonnet et al., 2001; de Dreuzy et al., 2001). Maximum and minimum fracture lengths are taken as $l_{\max }=20 \mathrm{~cm}$ and $l_{\min }=1 \mathrm{~cm}$, respectively. The orientation of the fractures and the positions of the fracture center are drawn from a uniform distribution, with fracture orientations being limited to angles between $30^{\circ}$ and $150^{\circ}$. This parametrization permits to obtain a backbone, that is, a connected fluid path within the fractured network, which allows for the flow of fluids from the left edge of the sample to the right. We consider a realization that exhibits two preferential fracture orientations in $\pm 30^{\circ}$ (Figure $6 \mathrm{~b}$ ), which emulates the preferential orientation of natural fractures with respect to a maximum principal compressive stress. All fractures crossing a sample's edge are continued on the opposite boundary, thus rendering the sample periodic (Figure 6d). The fracture density, which describes the relative area covered by fractures, is $3 \%$. The thickness of the fractures is considered to be constant and equal to $0.5 \mathrm{~mm}$. The physical properties of the sample's solid matrix and pore fluids are summarized in Table 1.

In order to explore the effects of partial saturation on the seismic signatures for the fractured medium described above, we simulate an IP process (see Section 2.3), in which $\mathrm{CO}_{2}$ displaces brine from the fractures. The $2 \mathrm{D}$ medium considered to perform the invasion simulations is composed by three repetitions of the probed sample, for which we 


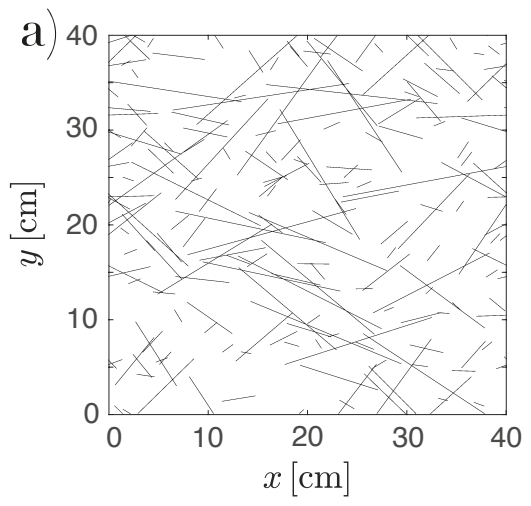

d) b)

All Fractures

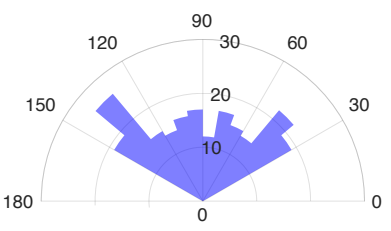

c) Backbone Fractures

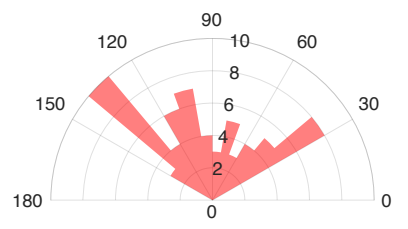

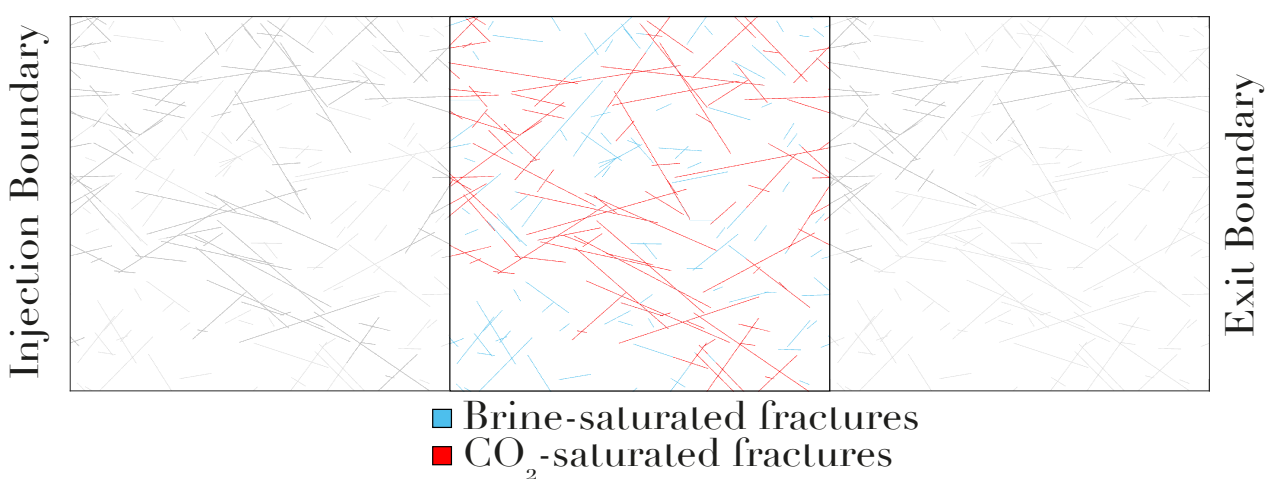

Figure 6. (a) Synthetic rock sample considered to analyze the seismic properties of stochastic partially saturated fractured media. Histograms of the fracture dips composing the (b) complete fracture network and (c) backbone. (d) Illustration of the saturation distribution within the fracture network resulting from an invasion percolation realization. Note that we employ an extended medium, which comprises three lateral repetitions of the probed medium, to perform the invasion. 
make use of the periodicity of the fracture network (Figure 6d). We define the invasion (left) and exit (right) boundaries (Figure 6d). Subsequently, the seismic response is studied considering the fluid distribution in the central region, thus avoiding boundary effects related to the IP procedure. As demanded by the IP procedure, we discretize the probed porous medium in a regular grid, where each cell $\Omega_{i j}$ has a local characteristic throat radius $r_{p, i j}$ and, hence, a particular entry pressure $p_{c, i j}^{e}$ (equation 10). We take the interfacial tension $\gamma=32 \times 10^{-3} \mathrm{~N} / \mathrm{m}$ and the contact angle $\beta=10^{\circ}$, which are characteristic values for the supercritical $\mathrm{CO}_{2}$-brine-quartz interface (e.g., Saraji et al., 2013). Recall that, in this work, we conceptualize fractures as regions of high porosity and permeability. This model has been proven to be acceptable with regards to the upscaled seismic properties (e.g., Quintal et al., 2016). Conversely, the structure at the microscopic scale, such as, irregular walls, contact areas, and grain infill, which is expected to influence the distribution of pore fluid phases during a capillary dominated flow process, is unknown to us. Therefore, we consider a uniformly distributed random assignment of the characteristic pore throat size value $10 \mu \mathrm{m}<r_{p, i j}<100 \mu \mathrm{m}$ within the cells comprising the fractures. The cells composing the background are not accessible to the invasion of $\mathrm{CO}_{2}$.

An example of a fluid distribution generated by this procedure along the probed fractured medium is presented in Figure 6d. It is important to remark here that, due to capillary effects, brine tends to remain present in those regions of the fracture network with high entry pressures (low $r_{p, i j}$ values). In order to account for the uncertainty associated with the random assignment of $r_{p, i j}$ values within the fractures, we explore the seismic response using a Monte Carlo analysis. For this, we consider 42 simulations of the IP process, using different seeds in each simulation for determining the characteristic pore throat radii $r_{p, i j}$ at each cell $\Omega_{i j}$. Then, we analyze the corresponding mean seismic response.

\subsection{Seismic Attenuation and Phase Velocity Dispersion}

When comparing the seismic response of the brine-saturated rock to the partially saturated scenarios resulting from the IP simulations, we observe that the P-wave attenuation due to FB-FPD decreases drastically (Figure 7a). This is expected, as seen in the previous section, because the presence of $\mathrm{CO}_{2}$ in a significant portion of the fracture network tends to diminish the generation of pressure gradients between fractures 

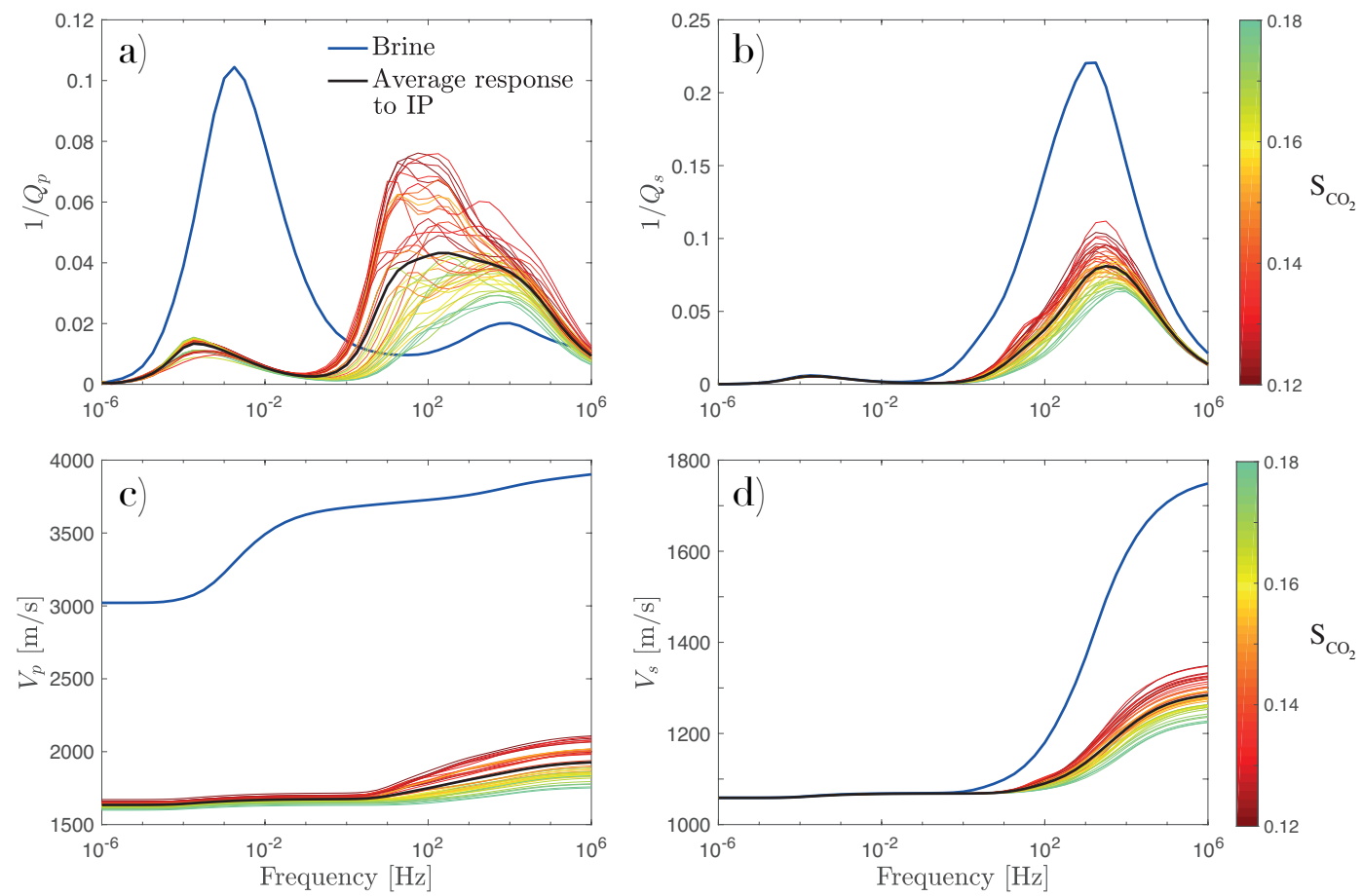

Figure 7. (a, b) Inverse quality factor and $(c, d)$ phase velocity dispersion as functions of frequency for vertically propagating P- and S-waves, respectively. We illustrate the behavior of 42 different IP realizations with the color scale denoting the overall $\mathrm{CO}_{2}$ saturation $\mathrm{S}_{\mathrm{CO}_{2}}$ of the rock sample for a given particular simulation. The blue and black solid lines illustrate the brinesaturated response and the average behavior for partial saturation based on all 42 IP simulations, respectively. 
and their embedding background. We observe that all partially saturated scenarios exhibit larger P-wave attenuation due to FF-FPD than that related to the fully brine-saturated state. This behavior, as seen in the numerical experiment analyzed in Section 3, is related to the presence of connected fractures that are saturated partly with $\mathrm{CO}_{2}$ and partly with brine. Furthermore, we note that the attenuation increases and the corresponding characteristic frequencies are shifted towards lower frequencies with decreasing $\mathrm{CO}_{2}$ saturation (Figures $7 \mathrm{a}$ and $7 \mathrm{c}$ ). This characteristic is associated to a FPD process occurring between $\mathrm{CO}_{2}$ - and brine-saturated regions within the fracture network, which will be further analyzed in section 4.3. This result is important and interesting, as it shows that the FF-FPD effects can produce strong attenuation in the seismic and sonic frequency bands in presence of partial saturation, even if they lie beyond the corresponding frequency bands under fully brine-saturated conditions. Note that P-wave attenuation characteristics associated with partially saturated scenarios exhibit maxima whose magnitudes are modulated by the overall $\mathrm{CO}_{2}$ saturation of the rock (Figure 7a). Also, the presence of $\mathrm{CO}_{2}$ significantly reduces the $\mathrm{P}$-wave phase velocity values and the corresponding dispersion (Figure 7c).

In the case of S-waves, we observe that FB-FPD effects tend to be negligible for the fully brine-saturated case and all partially saturated scenarios (Figure 7b and 7d). Also, we note that FF-FPD effects in partially saturated media produce lower $1 / Q_{s}$ values when compared to the fully brine-saturated rock (Figure 7b). These characteristics were also observed in Section 3, considering a simple fractured medium (Figure 5). Partial saturation generates contrasting behaviors in seismic attenuation for P- and S-waves due to FF-FPD. That is, $\mathrm{P}$-waves can exhibit an increase of the attenuation while $\mathrm{S}$-waves always exhibit a corresponding decrease due to this FPD process. The S-wave attenuation characteristics associated with partially saturated scenarios exhibit maxima whose magnitudes are modulated by the overall $\mathrm{CO}_{2}$ saturation of the rock. Smaller values of $S_{\mathrm{CO}_{2}}$ are associated to higher levels of attenuation and higher velocities. The characteristic frequency of the FF-FPD process remains virtually unchanged when comparing the fully brine-saturated and partially saturated scenarios, as S-waves are not particularly sensitive to spatial variations in the compressibility of the saturating fluids (i.e., patchy saturation). The presence of $\mathrm{CO}_{2}$ significantly reduces both the $\mathrm{S}$-wave phase velocity values, for sufficiently high frequencies, and the corresponding dispersion (Figure $7 d)$. 


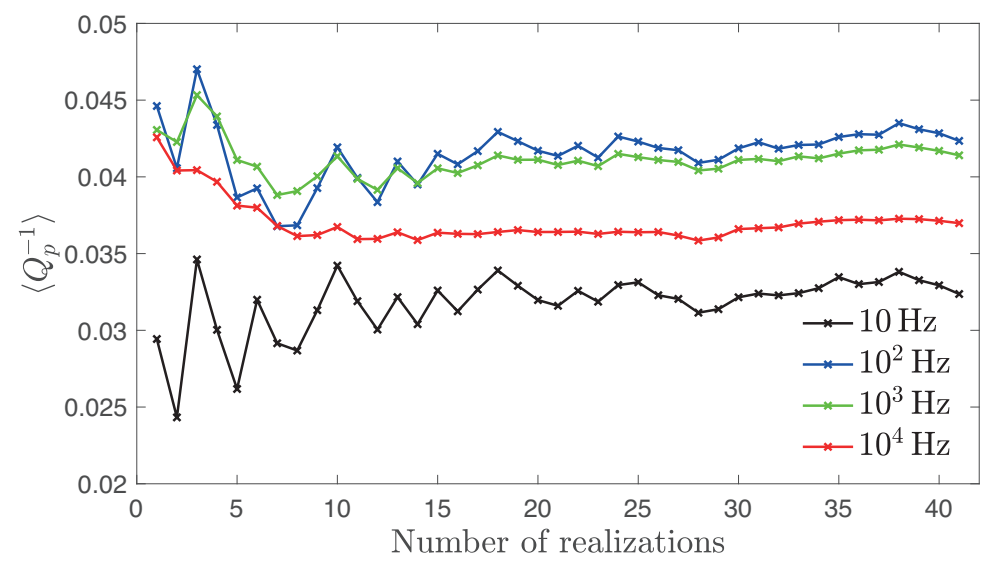

Figure 8. Mean value of $1 / Q_{p}$ for a vertical propagation as a function of the number of IP realizations for $10 \mathrm{~Hz}$ (black line), $10^{2} \mathrm{~Hz}$ (blue line), $10^{3} \mathrm{~Hz}$ (green line), and $10^{4} \mathrm{~Hz}$ (red line).

As mentioned above, we compute the mean behavior of the P- and S- wave seismic attenuation for partial saturation (black lines in Figure 7). Indeed, by considering a sufficiently large number of IP realizations, this behavior can be regarded as representative of the considered probed medium. We observe that the mean $1 / Q_{p}$ values tend to stabilize after approximately 30 simulations (Figure 8). This implies that the 42 simulations considered in this analysis are sufficient to obtain a representative behavior of the explored medium under partially saturated conditions.

The average $\mathrm{P}$ - and S-wave characteristics for partial saturation exhibit changes in the anisotropic response of the medium with respect to the fully brine-saturated state (Figure 9). Once again, we note that FB-FPD processes for the P-wave are hindered by the presence of $\mathrm{CO}_{2}$ in the fractures (Figures 9a and 9b). Attenuation due to FF-FPD presents peaks at incidence angles of approximately $35^{\circ}$ and $145^{\circ}$ for both the fully brinesaturated and the partially saturated responses. These angles are in agreement with the azimuthal location of the maxima in the polar fracture histogram (Figure 6). We also note that $\mathrm{P}$-wave attenuation due to FF-FPD increases for all incident angles under partially saturated conditions as compared with the fully brine-saturated rock, but there is a more pronounced attenuation peak for $145^{\circ}$ incidence. When analyzing FF-FPD processes associated with the S-wave propagation, we note that, compared to the fully brinesaturated rock (Figure 9c), attenuation values decrease for all angles in the presence of partial saturation (Figure 9d). 
a)

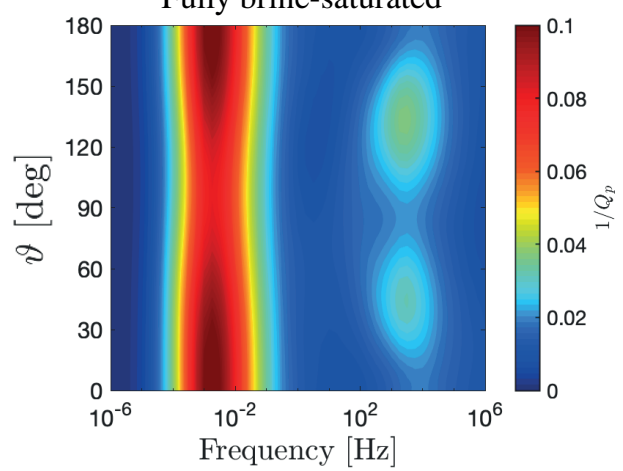

c)

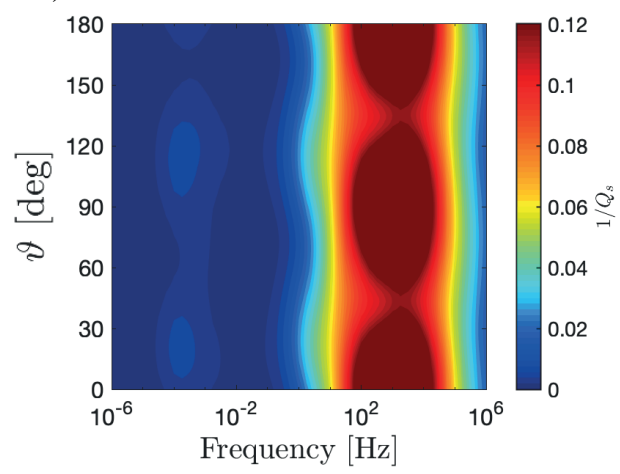

b) Brine- and $\mathrm{CO}_{2}$-saturated

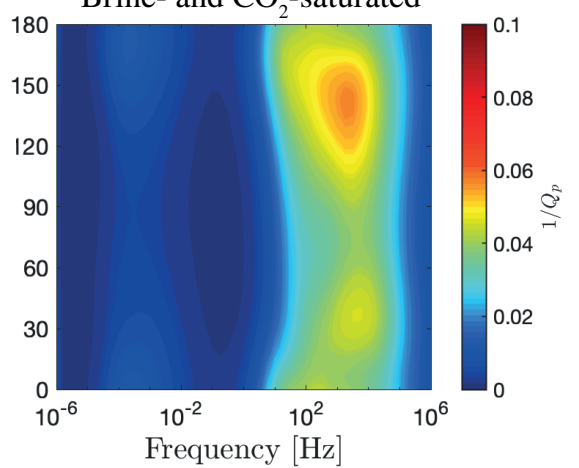

d)

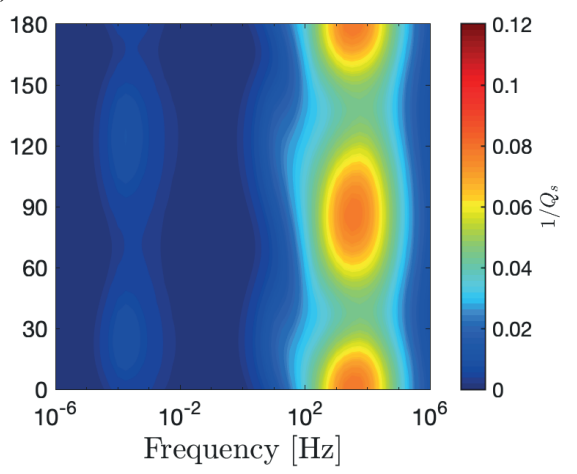

Figure 9. P-and S-wave attenuation as functions of frequency and incidence angle. Panels (a) and (c) show the seismic response when the rock sample is fully saturated with brine, while (b) and (d) depict the mean behaviors resulting from the IP procedure. 

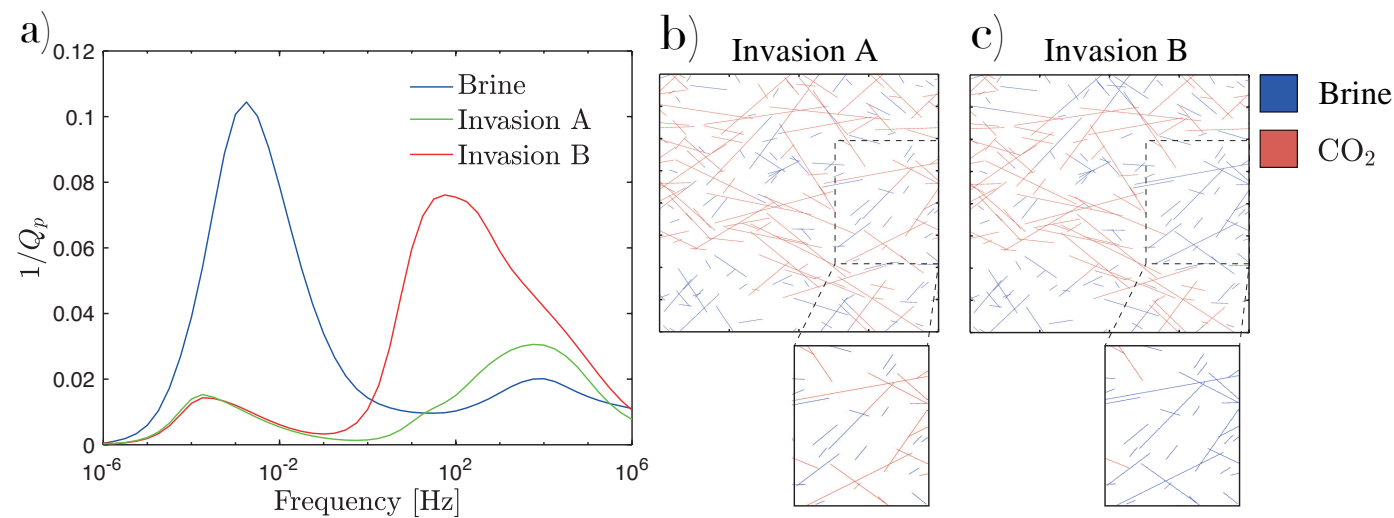

Figure 10. (a) Inverse quality factor for vertically traveling P-waves as a function of frequency for the brine-saturated (blue line) and two IP realisations, denoted as invasion A and invasion B, characterized by contrasting FF-FPD responses. Panels (b) and (c) show the corresponding fluid distributions.

\subsection{Effects of the Spatial Distribution of Fluids on the Seismic Signa- tures}

Even though the seismic attenuation and phase velocity dispersion curves for each IP realization present similar features, they exhibit variations. Interestingly, these changes are related to the $\mathrm{CO}_{2}$ saturation $S_{\mathrm{CO}_{2}}$ and to the spatial distribution of the two fluid phases. Due to the stochastic choice of local entry pressure values within the fractures, each invasion results in a different $\mathrm{CO}_{2}$ distribution throughout the fracture network. Whenever the properties of the fractures present a rather straight path for the $\mathrm{CO}_{2}$ to percolate through the rock sample, the resulting $S_{\mathrm{CO}_{2}}$ is relative small. Conversely, for those IP realisations that do not present such a direct "least resistance" path, the $\mathrm{CO}_{2}$ spreads across the backbone, resulting in larger overall $S_{\mathrm{CO}_{2}}$ values.

In order to analyze the effects of fluid distribution on seismic attenuation and phase velocity dispersion, we consider two IP realisations with contrasting seismic responses: invasions $\mathrm{A}$ and $\mathrm{B}$, which are characterized by presenting overall $\mathrm{CO}_{2}$ saturations $S_{\mathrm{CO}_{2}}^{A}=$ 0.16 and $S_{\mathrm{CO}_{2}}^{B}=0.13$, respectively. The P-wave attenuation associated with Invasion $\mathrm{B}$, for a wave traveling in the vertical direction, presents two superimposed peaks related to FF-FPD, which are located at $\sim 10^{2} \mathrm{~Hz}$ and $\sim 10^{4} \mathrm{~Hz}$ (Figure 10a). This characteristic is not present in the attenuation due to FF-FPD for Invasion A, which exhibits one attenuation peak at $\sim 10^{4} \mathrm{~Hz}$ (Figure 10a). By comparing the fluid distributions 
generated by both IP realisations (Figures 10b and 10c), we note that the fluid distribution resulting from Invasion B exhibits relatively large regions of the fracture network that are not invaded by $\mathrm{CO}_{2}$. Although not shown here for brevity, the pore fluid pressure fields associated with Invasion B indicate that, for a frequency of $10^{2} \mathrm{~Hz}$, fluid pressure gradients arise between regions of the fracture network that are brine-saturated and regions that are invaded with $\mathrm{CO}_{2}$. Such pressure gradients do not arise in Invasion A, which presents a more uniform distribution of $\mathrm{CO}_{2}$ across the backbone. Interestingly, the pressure gradients arising when the rock sample has a fluid distribution resulting from Invasion $\mathrm{B}$ generate $\mathrm{FF}$-FPD between regions of the fracture network that are $\mathrm{CO}_{2}$-saturated and connected regions that are brine-saturated. The scales at which this partially saturated FF-FPD process operates are larger than FF-FPD in fully brine-saturated conditions and, hence, we observe a second attenuation peak arising at $\sim 10^{2} \mathrm{~Hz}$ in Figure 10a for Invasion B. These results show that the seismic signatures are not only sensitive to the presence of a second and more compliant fluid phase but, also, they are sensitive to the spatial distribution of the two fluids within the fractured network.

The spatial distribution of the two fluids throughout the fracture network can also affect the anisotropic behavior of the medium. For a frequency of $100 \mathrm{~Hz}$, the less uniform fluid distribution resulting from Invasion B across the fracture network significantly increases the attenuation anisotropy when compared to the fluid distribution generated by Invasion A (Figure 11a). For reference, we also illustrate the mean behavior of all IP simulations and the fully brine-saturated case. For S-waves, invasion B also produces a more pronounced anisotropic response of $1 / Q_{s}$ than Invasion A (Figure 11b). Exploring the corresponding phase velocity responses, we note that the P- and S-wave anisotropy do not seem to present large changes as a result of such variations in the fluid distribution (Figures 11c and 11d). This characteristic of the P- and S-wave velocities, which is maintained for higher frequencies, evidences that even if the velocities are sensitive to the overall saturation level, the spatial distribution of fluids do not seem to affect significantly their anisotropic response.

\section{Discussion}

In this work, we have studied mesoscopic FPD effects in partially saturated fractured rocks. Two novel aspects are addressed: (i) the effects of heterogenous fluid distributions within a fracture network and (ii) the corresponding FF-FPD characteristics. 

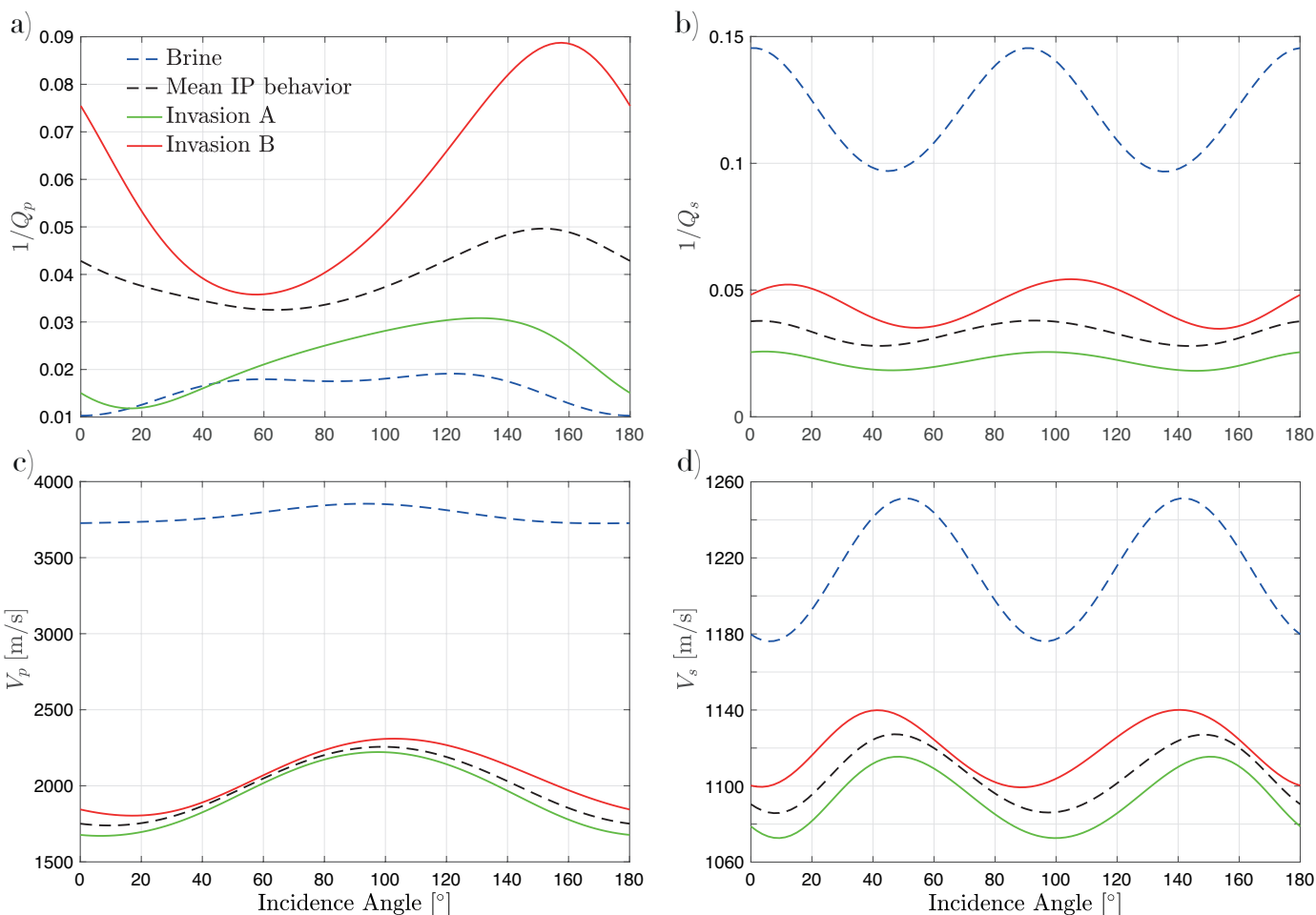

Figure 11. (a, b) Inverse quality factors and (c, d) seismic velocities as functions of incidence angle for the cases analyzed in Figure 10. We show the behavior for vertically traveling (a, c) Pand (b, d) S- waves for a frequency of $100 \mathrm{~Hz}$. 
The effects of partial saturation in FB-FPD have been previously studied by Kong et al. (2013) using an analytical solution for a set of aligned parallel 1D fractures. These authors observed that FB-FPD effects decrease when fractures that control the associated dissipation process are invaded by a more compliant fluid phase. Our results not only confirm this observation but, also prove that the reduction of FB-FPD effects can occur if fully brine-saturated fractures that control this process are connected to $\mathrm{CO}_{2^{-}}$ saturated fractures.

Several aspects of the seismic response of monosaturated rocks comprising complex fracture networks were analyzed by Hunziker et al. (2018). The authors explored the effects of fracture density, length distribution, and fracture connectivity for vertically propagating seismic waves. The results of this study are therefore complemented by ours, as we show that there are some characteristic signatures of seismic waves when a fracture network becomes partially saturated, such as the reduction of FB-FPD, and the possible increase of FF-FPD for P-waves. In addition, we showed that variations in the attenuation with incidence angle can be increased by highly heterogeneous pore fluid distributions within a fracture network.

It is important to remark that, due to numerical restrictions, our approach is limited to 2D samples and fractures are modeled considering constant apertures, which, in turn, results in fracture networks that exhibit a range of aspect ratios. Future works should address an extension to 3D and, also, the possibility of including different fracture apertures, which tend to be related to the corresponding fracture lengths. In this context, the entry pressure values within a fracture could be directly linked to its aperture. Finally, we focused on analyzing mesoscopic FPD effects and, thus, we ignored scattering effects and other dissipation mechanisms, such as, microscopic squirt flow and the intrinsic Biot mechanism (global flow), although they may be present for sufficiently high frequencies.

\section{Conclusion}

We analyzed seismic P- and S-wave attenuation and phase velocity dispersion characteristics as a function of frequency and incidence angle in a complex stochastic fracture network saturated to varying degrees by brine and $\mathrm{CO}_{2}$. On the one hand, our results show that dissipation due to FB-FPD is reduced due to the presence of $\mathrm{CO}_{2}$ in the 
fractured network. In this context, attenuation and dispersion curves are manly affected by the presence of $\mathrm{CO}_{2}$ in the fracture network, and not by the overall saturation. On the other hand, partial saturation at the mesoscopic scale can increase P-wave attenuation due to FF-FPD. However, S-wave attenuation is always reduced when $\mathrm{CO}_{2}$ invades the fractured rock. Furthermore, the magnitude of the attenuation and phase velocity due to FF-FPD, for both $\mathrm{P}$ - and $\mathrm{S}$ - waves, is modulated by the $\mathrm{CO}_{2}$ saturation of the sample. This characteristic behavior of P- and S-waves may help to detect and quantify the saturation of a second and more compliant pore fluid across regions of a fractured formation. We also observed changes in the seismic response associated with the spatial distribution of the two fluids within the fracture network. Particularly, we identified a novel FPD process, acting mainly on compressional waves, that takes place between $\mathrm{CO}_{2}$ saturated and brine-saturated regions of the fracture network. As a result of this process, the characteristic frequency of fracture-to-fracture fluid-pressure diffusion for $\mathrm{P}$-waves is shifted to lower values and the range of frequencies presenting relatively large attenuation values is broadened. Moreover, the angle dependence of attenuation is increased when the $\mathrm{CO}_{2}$ distribution within the fracture network is highly heterogeneous. This demonstrates that even if waves at seismic frequencies traveling through a brine-saturated fractured rock are not affected by wave-induced fluid flow, significant attenuation and velocity dispersion may arise for a corresponding partially saturated scenario.

\section{Acknowledgements}

S. G. S. thanks Nicolás D. Barbosa and Lucas X. Pimienta for their insightful comments during the early stages of this work. M. F. acknowledges gratefully the support of the Swiss National Science Foundation (SNSF), through the grant PZ00P2_180112. J. G. R. acknowledges the financial support received from the Agencia Nacional de Promoción Científica y Tecnológica of Argentina (PICT 2017-2976). This study was completed within the Swiss Competence Center for Energy Research-Supply of Electricity with support of Innosuisse. The data associated with this paper are available online from https://doi.org/10.5281/zenodo.3747519.

\section{References}

Amalokwu, K., Best, A. I., Sothcott, J., Chapman, M., Minshull, T., \& Li, X.-Y. 
(2014). Water saturation effects on elastic wave attenuation in porous rocks with aligned fractures. Geophys. J. Int., 197(2), 943-947.

Amalokwu, K., Chapman, M., Best, A. I., Minshull, T. A., \& Li, X.-Y. (2015). Water saturation effects on P-wave anisotropy in synthetic sandstone with aligned fractures. Geophys. J. Int., 202(2), 1088-1095.

Arts, R. J., Eiken, O., Chadwick, A., Zweigel, P., van der Meer, L., \& Zinszner, B. (2004). Monitoring of $\mathrm{CO}_{2}$ injected at Sleipner using time-lapse seismic data. Energy, 29, 1383-1392.

Ba, J., Xu, W., Fu, L.-Y., Carcione, J. M., \& Zhang, L. (2017). Rock anelasticity due to patchy saturation and fabric heterogeneity: A double double-porosity model of wave propagation. J. Geophys.Res.: Solid Earth, 122(3), 1949-1976.

Barbosa, N. D., Köpke, C., Caspari, E., Germán Rubino, J., Irving, J., \& Holliger, K. (2020). Impact of poroelastic effects on the inversion of fracture properties from AVOAz data in HTI media. Geophysics, 85(5), 1-48.

Bear, J. (1972). Dynamics of Fluids in Porous Media. New York: Am. Elsevier.

Biot, M. A. (1941). General theory of three-dimensional consolidation. J. Appl. Phys., 12, 155-164. doi: 10.1063/1.1712886

Biot, M. A. (1962). Mechanics of deformation and acoustic propagation in porous media. J. Appl. Phys., 33(4), 1482-1498. doi: 10.1063/1.1728759

Bonnet, E., Bour, O., Odling, N. E., Davy, P., Main, I., Cowie, P., \& Berkowitz, B. (2001). Scaling of fracture systems in geological media. Rev. Geophys., 39(3), $347-383$.

Brajanovski, M., Müller, T. M., \& Parra, J. O. (2010). A model for strong attenuation and dispersion of seismic P-waves in a partially saturated fractured reservoir. Sci. China Phys. Mech. Astron., 53(8), 1383-1387.

Caspari, E., Milani, M., Rubino, J. G., Muller, T. M., Quintal, B., \& Holliger, K. (2016). Numerical upscaling of frequency-dependent P-and S-wave moduli in fractured porous media. Geophys. Prospect., 64(4), 1166-1179.

Chapman, M. (2003). Frequency-dependent anisotropy due to meso-scale fractures in the presence of equant porosity. Geophys. Prospect., 51(5), 369-379.

Chapman, M. (2009). Modeling the effect of multiple sets of mesoscale fractures in porous rock on frequency-dependent anisotropy. Geophysics, 74(6), D97D103. 
de Dreuzy, J.-R., Davy, P., \& Bour, O. (2001). Hydraulic properties of twodimensional random fracture networks following a power law length distribution: 1. Effective connectivity. Water Resour. Res., 37(8), 2065-2078.

Farina, B., Poletto, F., Mendrinos, D., Carcione, J. M., \& Karytsas, C.

(2019) Seismic properties in conductive and convective hot and super-hot geothermal systems. Geothermics, 82, 16-33.

Favino, M., Hunziker, J., Caspari, E., Quintal, B., Holliger, K., \& Krause, R. (2019). Fully-automated adaptive mesh refinement for media embedding complex heterogeneities: application to poroelastic fluid pressure diffusion. Comput. Geosci.. doi: 10.1007/s10596-019-09928-2

Gurevich, B., Brajanovsky, M., Galvin, R. J., Müller, T. M., \& Toms-Stewart, J. (2009). P-wave dispersion and attenuation in fractured and porous reservoirs- poroelasticity approach. Geophys. Prospect., 57, 225-237. doi: 10.1111/j.1365-2478.2009.00785.x

Han, T., Gurevich, B., Fu, L.-Y., Qi, Q., Wei, J., \& Chen, X. (2019). Combined effects of pressure and water saturation on the seismic anisotropy in artificial porous sandstone with aligned fractures. J. Geophys. Res.: Solid Earth, e2019JB019091.

Hardisty, P. E., Wheater, H. S., Birks, D., \& Dottridge, J. (2003). Characterization of LNAPL in fractured rock. Q. J. Eng. Geol. Hydroge., 36(4), 343-354.

Hunt, L., Reynolds, S., Brown, T., Hadley, S., Downton, J., \& Chopra, S. (2010). Quantitative estimate of fracture density variations in the Nordegg with azimuthal AVO and curvature: A case study. Lead. Edge, 29(9), 1122-1137.

Hunziker, J., Favino, M., Caspari, E., Quintal, B., Rubino, J. G., Krause, R., \& Holliger, K. (2018). Seismic attenuation and stiffness modulus dispersion in porous rocks containing stochastic fracture networks. J. Geophys. Res., 123(1), 125-143.

Jin, Z., Chapman, M., \& Papageorgiou, G. (2018). Frequency-dependent anisotropy in a partially saturated fractured rock. Geophys. J. Int., 215(3), 1985-1998.

Karpyn, Z., Grader, A., \& Halleck, P. (2007). Visualization of fluid occupancy in a rough fracture using micro-tomography. J. Colloid Interf. Sci., 307(1), 181187.

Kong, L., Gurevich, B., Müller, T. M., Wang, Y., \& Yang, H. (2013). Effect of frac- 
ture fill on seismic attenuation and dispersion in fractured porous rocks. Geophys. J. Int., 195(3), 1679-1688.

Lenormand, R., Touboul, E., \& Zarcone, C. (1988). Numerical models and experiments on immiscible displacements in porous media. J. Fluid Mech., 189, 165187.

Lumley, D. E. (2001). Time-lapse seismic reservoir monitoring. Geophysics, 66(1), $50-53$.

Masson, Y. (2016). A fast two-step algorithm for invasion percolation with trapping. Comput and Geosci., 90, 41-48.

Masson, Y., \& Pride, S. R. (2007). Poroelastic finite difference modeling of seismic attenuation and dispersion due to mesoscopic-scale heterogeneity. J. Geophys. Res., 112, B03204.

Masson, Y., \& Pride, S. R. (2014). A fast algorithm for invasion percolation. Transp. Porous Med., 102(2), 301-312.

Papageorgiou, G., \& Chapman, M. (2017). Wave-propagation in rocks saturated by two immiscible fluids. Geophys. J. Int., 209(3), 1761-1767.

Quintal, B., Jänicke, R., Rubino, J. G., Steeb, H., \& Holliger, K. (2014). Sensitivity of S-wave attenuation to the connectivity of fractures in fluid-saturated rocks. Geophysics, 79(5), WB15-WB24.

Quintal, B., Rubino, J. G., Caspari, E., \& Holliger, K. (2016). A simple hydromechanical approach for simulating squirt-type flow. Geophysics, 81(4), D335D344.

Rubino, J. G., Caspari, E., Müller, T. M., \& Holliger, K. ～(2017). Fracture connectivity can reduce the velocity anisotropy of seismic waves. Geophys. J. Int., 210(1), 223-227.

Rubino, J. G., Caspari, E., Müller, T. M., Milani, M., Barbosa, N. D., \& Holliger, K. (2016). Numerical upscaling in 2-D heterogeneous poroelastic rocks: Anisotropic attenuation and dispersion of seismic waves. J. Geophys. Res. $121(9), 6698-6721$.

Rubino, J. G., Guarracino, L., Müller, T. M., \& Holliger, K. ～(2013). Do seismic waves sense fracture connectivity? Geophys. Res. Lett., 40(4), 692-696.

Rubino, J. G., \& Holliger, K. (2012). Seismic attenuation and velocity dispersion in heterogeneous partially saturated porous rocks. Geophys. J. Int., 188, 1088- 
1102. doi: $10.1111 / \mathrm{j} .1365-246 X .2011 .05291 . x$

Rubino, J. G., Müller, T. M., Guarracino, L., Milani, M., \& Holliger, K.

(2014). Seismoacustic signatures of fracture connectivity.

J. Geophys. Res., 119, 2252-2271. doi: 10.1002/2013JB010567

Rubino, J. G., Velis, D. R., \& Sacchi, M. D. (2011). Numerical analysis of waveinduced fluid flow effects on seismic data: Application to monitoring of $\mathrm{CO}_{2}$ storage at the Sleipner field. J. Geophys. Res., 116, 1088-1102. doi: 10.1029/2010JB007997

Rueger, A., \& Tsvankin, I. (1997). Using AVO for fracture detection: Analytic basis and practical solutions. Lead. Edge, 16 (10), 1429-1434.

Saraji, S., Goual, L., Piri, M., \& Plancher, H. (2013). Wettability of supercritical carbon dioxide/water/quartz systems: Simultaneous measurement of contact angle and interfacial tension at reservoir conditions. Langmuir, 29(23), 68566866 .

Smith, S., \& Snieder, R. (2010). Seismic modeling and analysis of a prototype heated nuclear waste storage tunnel, Yucca Mountain, Nevada. Geophysics, $75(1), \mathrm{T} 1-\mathrm{T} 8$.

Solazzi, S. G., Guarracino, L., Rubino, J. G., \& Holliger, K. (2019). Saturation hysteresis effects on the seismic signatures of partially saturated heterogeneous porous rocks. J. Geophys. Res. Solid Earth, 124(11), 11316-11335. doi: 10.1029/2019JB017726

Solazzi, S. G., Guarracino, L., Rubino, J. G., Müller, T. M., \& Holliger, K. (2017). Modeling forced imbibition processes and the associated seismic attenuation in heterogeneous porous rocks. J. Geophys. Res. Solid Earth, 122(11), 90319049.

Solazzi, S. G., Rubino, J. G., Müller, T. M., Milani, M., Guarracino, L., \& Holliger, K. (2016). An energy-based approach to estimate seismic attenuation due to wave-induced fluid flow in heterogeneous poroelastic media. Geophys. J. Int., 207(2), 823-832.

Vinci, C., Renner, J., \& Steeb, H. (2014). On attenuation of seismic waves associated with flow in fractures. Geophys. Res. Lett., 41(21), 7515-7523.

Wilkinson, D., \& Willemsen, J. F. (1983). Invasion percolation: a new form of percolation theory. J. Phys. A, 16(14), 3365. 